\title{
Effect of Virtual Reality on Cognitive Dysfunction in Patients With Brain Tumor
}

\author{
Seoyon Yang, MD, Min Ho Chun, MD, PhD, Yu Ri Son, MD
}

Department of Rehabilitation Medicine, Asan Medical Center, University of Ulsan College of Medicine, Seoul, Korea

Objective To investigate whether virtual reality (VR) training will help the recovery of cognitive function in brain tumor patients.

Methods Thirty-eight brain tumor patients (19 men and 19 women) with cognitive impairment recruited for this study were assigned to either VR group ( $n=19$, IREX system) or control group ( $n=19)$. Both VR training (30 minutes a day for 3 times a week) and computer-based cognitive rehabilitation program (30 minutes a day for 2 times) for 4 weeks were given to the VR group. The control group was given only the computer-based cognitive rehabilitation program (30 minutes a day for 5 days a week) for 4 weeks. Computerized neuropsychological tests (CNTs), Korean version of Mini-Mental Status Examination (K-MMSE), and Korean version of Modified Barthel Index (K-MBI) were used to evaluate cognitive function and functional status.

Results The VR group showed improvements in the K-MMSE, visual and auditory continuous performance tests (CPTs), forward and backward digit span tests (DSTs), forward and backward visual span test (VSTs), visual and verbal learning tests, Trail Making Test type A (TMT-A), and K-MBI. The VR group showed significantly $(\mathrm{p}<0.05)$ better improvements than the control group in visual and auditory CPTs, backward DST and VST, and TMT-A after treatment.

Conclusion VR training can have beneficial effects on cognitive improvement when it is combined with computerassisted cognitive rehabilitation. Further randomized controlled studies with large samples according to brain tumor type and location are needed to investigate how VR training improves cognitive impairment.

Keywords Brain tumors, Cognition, Virtual reality therapy

Received June 10, 2014; Accepted August 18, 2014

Corresponding author: Min Ho Chun

Department of Rehabilitation Medicine, Asan Medical Center, University of Ulsan College of Medicine, 88 Olympic-ro 43-gil, Songpa-gu, Seoul 138-736, Korea

Tel: +82-2-3010-3800, Fax: +82-2-3010-6964, E-mail: mhchun@amc. seoul.kr

(c) This is an open-access article distributed under the terms of the Creative Commons Attribution Non-Commercial License (http://creativecommons. org/licenses/by-nc/3.0) which permits unrestricted noncommercial use, distribution, and reproduction in any medium, provided the original work is properly cited.

Copyright ( 2014 by Korean Academy of Rehabilitation Medicine

\section{INTRODUCTION}

Many brain tumor patients suffer from cognitive impairment and struggle to deal with daily life activities $[1,2]$. Symptoms caused by brain tumor, such as fatigue, lethargy, and cognitive impairment, are associated with distressed mood, resulting in reduced quality of life. Cognitive deficits in brain tumor patients are usually mild and diffuse which are different from those produced by strokes [1]. Cognitive impairment can be caused by the tumor itself or by cancer treatment, such as surgery, ra- 
diotherapy, chemotherapy, immunotherapy, and antiepileptic drugs $[1,3]$.

Various studies using virtual reality (VR) programs have suggested that VR may be helpful to improve cognitive function [4-8]. VR programs consist of real-time and three-dimensional environment that makes patients feel that they are participating in real-time situations. Patients hospitalized eventually adapt to the hospital environment, which hinders their adjustment to the outside world following hospital discharge. It may be especially hard for patients with cognitive impairment to deal with the real environment. VR training provides patients with virtual environment that they might experience in real life, which can mitigate these challenges.

Previous studies have shown the benefits of VR training. For example, VR training has been found to effectively improve memory and problem-solving skills in patients with Alzheimer disease [9], Parkinson disease [4,10], and in elderly people with mild cognitive impairment $[5,11]$. VR could also aid the recovery of stroke patients with upper extremity impairment [12] or unilateral neglect [13]. VR training and computer-assisted cognitive rehabilitation performed together were found to be more effective than computer-assisted cognitive rehabilitation alone, with VR training having additional benefits for cognitive function recovery in stroke patients [14].

To our knowledge, however, no previous study has shown the effectiveness of VR training in brain tumor patients who have cognitive impairment. The aim of this study was to investigate whether the effect of VR training and computer-assisted cognitive rehabilitation performed together was better than computer-assisted cognitive rehabilitation alone for these patients.

\section{MATERIALS AND METHODS}

\section{Study subjects}

The inclusion criteria for this study were 1) patients who had been diagnosed with a brain tumor for the first time and received treatments, such as surgery, radiotherapy, or chemotherapy; 2) patients who had been stabilized medically, neurologically, and surgically; 3 ) patients who had been transferred to the Department of Rehabilitation Medicine at Asan Medical Center for the treatment. All our enrolled patients had cognitive impairment with scores ranging from 10 to 26 based on the Korean version of the Mini-Mental Status Examination (K-MMSE), considering that patients with scores less than 10 might not be able to follow instructions to participate in our study. All included patients were more than 20 years old and agreed to participate. Exclusion criteria were 1) patients who had apraxia, neglect, or aphasia resulting in their inability to undergo proper cognitive evaluation were excluded; 2) patients who could not recognize objects presented on a computer screen due to visual impairment; $3)$ patients who could not receive cognitive rehabilitation 5 times a week due to other medical reasons; 4) patients who were unable to follow program instructions, and 5) patients with poor sitting balance who could not sit on a chair.

Included patients were randomly assigned into either VR group or control group. The VR group received both computer-assisted cognitive rehabilitation and VR training. The control group was given only the computer-assisted cognitive rehabilitation. The VR group received VR training 3 times a week for 30 minutes and computer-assisted cognitive rehabilitation 2 times a week for $30 \mathrm{~min}$ utes [14]. The control group received computer-assisted cognitive rehabilitation 5 times a week for 30 minutes. Both groups participated in these programs for a total of 4 weeks. Physical therapy and occupational therapy of the same intensity were given to all participants.

\section{Training programs}

IREX system (Vivid Group, Toronto, Canada) was used for VR training. The system consists of a monitor, video camera, virtual objects, and data gloves that are used to recognize the movement of patients. Out of $20 \mathrm{VR}$ programs, we selected 5 programs for our study [14]. Each program was performed for 6 minutes with a 1-minute break between programs (Fig. 1). Patients were encouraged to use the affected arm to participate in the programs. However, if it was impossible due to weakness, they were allowed to use the unaffected arm. Patients sat on a wheelchair when participating in the VR training. An occupational therapist guided the patients through the programs. The first program was 'Conveyor'. In this program, conveyor belts containing boxes appeared on the screen from the left or right side. Patients were asked to move the boxes from one side to the other. The second program was 'Coconuts'. From the top of the screen, coconuts fell down and patients tried to catch them and 

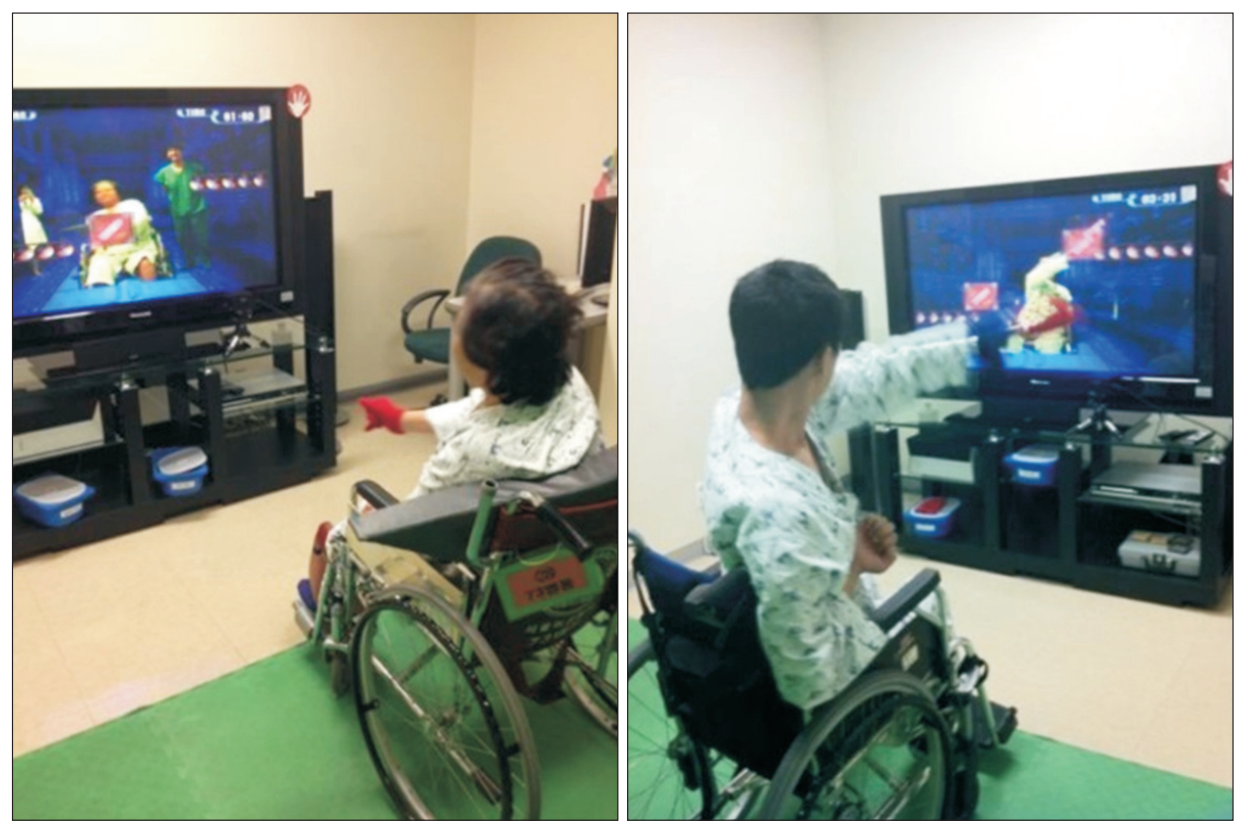

Fig. 1. Virtual reality programs on the IREX system (Vivid Group, Toronto, Canada).

put them into a basket. The third program was 'Bird and Balls. Balls came from various directions on the screen. When patients tried to grab them, they burst and changed into birds. The fourth program was called 'Soccer'. Soccer balls appeared on the screen and patients tried to stop the balls from entering the net, just like goalkeepers. The fifth program was called the 'Juggler'. Balls appeared on the screen simultaneously. Patients tried to hit the balls to move them upward to avoid dropping the balls on the floor. In each program, various kinds of background music were played. Scores achieved by patients were displayed on the screen after each program. The velocity, angle, and distance of objects were adjusted according to the levels of difficulty and patient condition.

ComCog (MaxMedica Inc., Seoul, Korea) was used for computer-assisted cognitive rehabilitation. This system contained programs that were designed to enhance attention and memory skills. The attention program consisted of three courses at beginner, intermediate, and advanced levels. The program included attention training, attention discrimination training, visual perception training, auditory perception training, continuous attention training, attention integration training, and emotion training. The level of training was determined based on task difficulty and patient condition. Memory programs also consisted of three courses at beginner, intermediate and advanced levels. The program included simple spatial memory training, simple recognition memory training, sequential recall memory training, sequential verbal recall memory training, associated recall memory training, verbal categorization memory training, and integrated memory training. Verbal/non-verbal tasks and sequential/non-sequential tasks were given to patients.

\section{Evaluation method}

Before the treatment, a computerized neuropsychological test (CNT, MaxMedica Inc.) was given to patients to evaluate their cognitive status. The CNT included visual and auditory continuous performance tests (CPTs) to evaluate continuous concentration on visual and auditory stimuli. A word-color test was used to evaluate selective attention. Forward and backward digit span tests (DSTs) and verbal learning tests were used to evaluate verbal memory. Forward and backward visual span tests (VSTs) and visual learning tests were used to assess spatial memory.

In verbal learning tests, patients were provided 15 different words. The number of words they first recalled was scored. The same words were repeated 5 times and the words they recalled were scored again. After 20 minutes, patients were asked to remember the words that they had heard and the words they remembered were scored once again. Subsequently, 30 different words, including the ones that were previously given, were shown on the computer screen and the patients were asked to choose the words that they remembered. 
As for visual learning tests, 15 different figures were sequentially shown. Thirty figures including the 15 figures that were previously presented were shown again and patients were asked to choose the 15 figures that were first shown. The same figures were shown again 5 times and patients chose the ones they knew. After 20 minutes, patients were asked to choose them again. All 30 figures were shown again and patients were asked to choose the previous 15 figures. The results were scored and recorded.

The Trail Making Test type A (TMA-A) was used to assess visual motor coordination. Patients drew lines to connect 25 circles. Each circle contained numbers from 1 to 25. Patients were asked to connect the lines in numerical order. When patients finished connecting these circles, the total amount of time needed to connect all circles was recorded.

Korean version of Modified Barthel Index (K-MBI) was used to evaluate activities of daily living function. This evaluation was performed by occupational therapists. Patients were re-evaluated after 4 weeks of rehabilitation treatment.

\section{Statistical analysis}

Mann-Whitney test or chi-square test was used to test
Table 1. Demographic characteristics of patients used in this study

\begin{tabular}{|ccc}
\hline & $\begin{array}{c}\text { VR group } \\
(\mathbf{n}=19)\end{array}$ & $\begin{array}{c}\text { Control group } \\
(\mathbf{n}=19)\end{array}$ \\
\hline Sex (male:female) & $9: 10$ & $10: 9$ \\
\hline Age (yr) & $47.9 \pm 14.5$ & $52.9 \pm 14.0$ \\
\hline Side of lesion & & \\
\hline Right & $8(42.1)$ & $9(47.4)$ \\
\hline Left & $11(57.9)$ & $10(52.6)$ \\
\hline Lesion location & & \\
\hline Frontal lobe & $6(31.6)$ & $7(36.8)$ \\
\hline Parietal lobe & $5(26.3)$ & $5(26.3)$ \\
\hline Temporal lobe & $1(5.3)$ & $2(10.5)$ \\
\hline Cerebellum & $3(15.8)$ & $2(10.5)$ \\
\hline Other sites & $4(21.0)$ & $3(15.8)$ \\
\hline Types of tumor & & \\
\hline Meningioma & $3(15.8)$ & $7(36.8)$ \\
\hline Glioblastoma & $2(10.5)$ & $3(15.8)$ \\
\hline Metastasis & $3(15.8)$ & $3(15.8)$ \\
\hline Astrocytoma & $2(10.5)$ & $0(0)$ \\
\hline Others & $9(47.4)$ & $6(31.6)$ \\
\hline
\end{tabular}

Values are presented as mean \pm standard deviation or number (\%).

VR, virtual reality.

Table 2. Changes after treatment in the VR group or the control group

\begin{tabular}{lcccccc}
\hline & \multicolumn{3}{c}{ VR group } & \multicolumn{3}{c}{ Control group } \\
\cline { 2 - 7 } & Before & After & p-value & Before & After & p-value \\
\hline VCPT $(\mathrm{sec})$ & $0.6 \pm 0.2$ & $0.5 \pm 0.1$ & $<0.01^{*}$ & $0.5 \pm 0.2$ & $0.4 \pm 0.2$ & 0.19 \\
ACPT $(\mathrm{sec})$ & $0.7 \pm 0.2$ & $0.6 \pm 0.1$ & $<0.01^{*}$ & $0.6 \pm 0.2$ & $0.5 \pm 0.2$ & $<0.01^{* *}$ \\
\hline WCW & $14.5 \pm 12.3$ & $12.7 \pm 10.0$ & 0.06 & $15.8 \pm 15.7$ & $13.4 \pm 12.8$ & 0.84 \\
\hline FDST & $4.8 \pm 1.9$ & $5.7 \pm 1.9$ & $<0.01^{*}$ & $4.8 \pm 1.8$ & $5.3 \pm 1.7$ & 0.10 \\
\hline BDST & $3.0 \pm 1.1$ & $4.4 \pm 1.6$ & $<0.01^{*}$ & $3.0 \pm 1.2$ & $3.3 \pm 1.5$ & 0.17 \\
FVST & $4.0 \pm 1.2$ & $4.9 \pm 1.3$ & $<0.01^{*}$ & $3.4 \pm 1.7$ & $3.9 \pm 1.7$ & $<0.01^{* *}$ \\
BVST & $3.0 \pm 1.0$ & $4.3 \pm 1.4$ & $<0.01^{*}$ & $2.4 \pm 1.2$ & $3.1 \pm 1.7$ & 0.27 \\
ViLT-R & $34.8 \pm 17.6$ & $46.7 \pm 13.9$ & $<0.01^{*}$ & $34.63 \pm 18.9$ & $41.0 \pm 20.2$ & $<0.01^{* *}$ \\
\hline VeLT-R & $26.1 \pm 15.7$ & $34.6 \pm 18.2$ & $<0.01^{*}$ & $23.2 \pm 13.4$ & $31.4 \pm 15.7$ & $<0.01^{*}$ \\
\hline TMT-A (sec) & $137.9 \pm 109.2$ & $70.2 \pm 68.8$ & $<0.01^{*}$ & $157.2 \pm 117.5$ & $132.7 \pm 113.1$ & 0.05 \\
\hline K-MBI & $43.4 \pm 26.6$ & $73.7 \pm 3.6$ & $<0.01^{*}$ & $43.2 \pm 29.1$ & $65.9 \pm 23.5$ & $<0.01^{*}$ \\
\hline MMSE & $19.8 \pm 3.8$ & $25.0 \pm 3.6$ & $<0.01^{*}$ & $21.0 \pm 4.6$ & $24.3 \pm 4.2$ & $<0.01^{*}$ \\
\hline
\end{tabular}

Values are presented as mean \pm standard deviation.

VR, virtual reality; VCPT, visual continuous performance test; ACPT, auditory continuous performance test; WCW, word of color word in word-color test; FDST, forward digit span test; BDST, backward digit span test; FVST, forward visual span test; BVST, backward visual span test; ViLT-R, visual learning test-recognition; VeLT-R, verbal learning test-recognition; TMT-A, Trail Making Test-type A; K-MBI, Korean version of Modified Barthel Index; K-MMSE, Korean version of the Mini-Mental Status Examination.

${ }^{*} \mathrm{p}<0.05$, Wilcoxon signed-ranks test. 
the homogeneity between the two groups. Changes shown before and after the treatments were analyzed using Wilcoxon signed-rank test in both groups. Mann-Whitney test was used to compare the differences in results in both groups. SPSS ver. 14.0 (SPSS Inc., Chicago, IL, USA) was used for all statistical analysis. A 95\% confidence interval and $\mathrm{p}<0.05$ indicated statistical significance.

\section{RESULTS}

A total of 19 patients with a mean age of $47.9 \pm 14.5$ years were enrolled in the VR group. In the control group, 19 patients with a mean age of $52.9 \pm 14.0$ years were enrolled. There were no statistically significant differences in age, sex, number of patients, duration of disease, location of lesion, or method of treatment between the two groups. All patients were not affected by medications known to have beneficial effects on cognitive impairment, such as methylphenidate, amantadine, donepezil, and other AChE inhibitors.

In the VR group, 8 patients had the brain lesions at the right side whereas 11 patients had the lesions at the left side. In the control group, 9 patients had the lesions at the right side whereas 10 patients had the lesions at the left side. In the VR group, most lesions were located in either frontal $(n=6)$ or parietal $(n=5)$ areas of the brain. In the control group, most lesions were located in either frontal $(n=7)$ or parietal $(n=5)$ areas of the brain. The rest of the lesions were located in other areas of the brain, such as temporal lobe, sellar region, or cerebellum (Table 1).

In the VR group after the treatment, there were statistically significant $(\mathrm{p}<0.05)$ improvements in the visual and auditory CPTs, forward and backward DSTs, forward and backward VSTs, verbal and visual learning tests, and TMT-A. In the control group after the treatment, statistically significant $(\mathrm{p}<0.05)$ improvements were observed in the auditory CPT, visual and verbal learning tests, and forward VST. Both K-MMSE and K-MBI showed improvements in both groups after the treatment (Table 2). The visual and auditory CPTs, backward DST, backward VST, and TMA-A showed a significantly $(\mathrm{p}<0.05)$ better improvement in the VR group than in the control group (Table 3).

\section{DISCUSSION}

As life expectancies of brain tumor patients are increas-
Table 3. Comparison of rehabilitation effect between the VR group and the control group

\begin{tabular}{lccc}
\hline & VR group & Control group & p-value \\
\hline$\Delta$ VCPT (sec) & $-0.2 \pm 0.2$ & $-0.0 \pm 0.2$ & $<0.01^{*}$ \\
$\Delta$ ACPT (sec) & $-0.2 \pm 0.2$ & $-0.0 \pm 0.1$ & $0.04^{*}$ \\
$\Delta$ WCW & $-1.8 \pm 3.9$ & $-2.4 \pm 5.3$ & 0.29 \\
$\Delta$ FDST & $0.9 \pm 1.2$ & $0.5 \pm 1.9$ & 0.25 \\
$\Delta$ BDST & $1.4 \pm 1.2$ & $0.3 \pm 0.8$ & $0.04^{*}$ \\
$\Delta$ FVST & $0.9 \pm 0.9$ & $0.5 \pm 1.4$ & 0.20 \\
$\Delta$ BVST & $1.4 \pm 1.2$ & $0.7 \pm 1.2$ & $0.03^{*}$ \\
$\Delta$ ViLT-R & $11.9 \pm 9.6$ & $6.4 \pm 9.8$ & 0.11 \\
$\Delta$ VeLT-R & $8.5 \pm 8.1$ & $8.3 \pm 9.4$ & 0.46 \\
$\Delta$ TMT-A (sec) & $-67.7 \pm 74.6$ & $-24.5 \pm 59.5$ & $0.02^{*}$ \\
$\Delta$ K-MBI & $30.5 \pm 31.2$ & $22.7 \pm 18.6$ & 0.64 \\
$\Delta$ MMSE & $5.3 \pm 3.2$ & $3.3 \pm 2.3$ & 0.06 \\
\hline
\end{tabular}

Values are presented as mean \pm standard deviation.

VR, virtual reality; VCPT, visual continuous performance test; ACPT, auditory continuous performance test; WCW, word of color word in word-color test; FDST, forward digit span test; BDST, backward digit span test; FVST, forward visual span test; BVST, backward visual span test; ViLT-R, visual learning test-recognition; VeLT$\mathrm{R}$, verbal learning test-recognition; TMT-A, Trail Making Test-type A; K-MBI, Korean version of Modified Barthel Index; K-MMSE, Korean version of the Mini-Mental Status Examination.

${ }^{*} \mathrm{p}<0.05$, Mann-Whitney test.

ing with the advance of treatment, interest into quality of life is also growing [1]. Intact cognitive function is necessary to maintain quality of survival and restore patients' independence for activities of daily living. Tumor, tumor progression, and neurological complications can induce cognitive deficits $[1,15,16]$ in brain tumor patients who often have decreased quality of life.

Unlike patients with stroke who suffer from site-specific deficits, patients with brain tumor, such as glioma, are known to have more global cognitive deficits, possibly due to the infiltration of diffuse growth of tumor cells [17]. Previous studies $[1,3]$ have explained that surgery and perioperative injuries could cause neurological deficits by damaging normal surrounding tissues. Chemotherapy also has adverse effects on cognitive functioning and radiotherapy, causing disruption to the blood-brain barrier by demyelination and vascular damage, such as vasogenic edema. Cognitive deficits in brain tumor patients can be unpredictable and hard to recover. In a study of fractionated whole brain radiation with brain metastases, 
a $91 \%$ incidence of baseline cognitive impairment correlated with tumor volume was reported [18]. Treatments for brain tumor patients have been targeted at cognitive domains, such as memory, attention, verbal learning, problem solving, frontal lobe executive functions, and information processing. Assessing cognitive functions specifically including these domains with a battery of tests is important [2].

For treating cognitive dysfunction, computerized interventions can be used effectively in clinical settings [3]. Computerized interventions can provide repetitive training, performance-related feedback, and motivation to patients [19]. Computer games can train cognitive functions, especially attention and executive functions. When they are combined with physical exercise, both sensorymotor function and cognitive inputs are required in the virtual environment $[3,20]$. In VR environments, patients are given multiple sensory modalities to interact with images and virtual objects in real-time [21]. VR has several advantages over conventional rehabilitation therapy. The virtual environments help patients increase their sense of autonomy and independence by giving patients the opportunity to explore independently. In addition, virtual environments can be delivered consistently. Since performances are recorded, patients can accurately and visibly compare their performances over time [22]. In chronic stroke patients, VR training has been shown to induce reorganization of sensorimotor cortex [23].

The results of our study suggest that VR could increase the motivation of brain tumor patients to actively participate in the programs. This increased motivation resulted in significant improvements in visual and auditory attention and short-term visuospatial memory when compared to the control group. It seems that VR is helpful for brain tumor patients who have cognitive impairment, especially in improving attention and short-term memory. The contribution of VR to this improvement could be explained that VR consists of programs that are interesting and enjoyable. Thus, patients who lack interest in traditional exercise program might be more willing to participate in VR training. When patients actively try to get involved in the programs, they concentrate on finishing the tasks given to them. This increased level of involvement can trigger their thinking processes. Patients will try to orientate, concentrate, analyze, and perform appropriate responses to the visual cues [24]. The 'gaming' factors in VR systems allow patients to enhance motivation and increase their participation, which can activate brain neurotransmitter pathways, such as those of cholinergic and dopaminergic systems. This increased participation has been shown to improve attention and memory in the elderly [25]. Patients are focused on achieving goals within the game [26], which is important because brain tumor patients who suffer from fatigue and poor motivation need to participate in rehabilitation programs that are interesting and motivating.

In a previous study [27], VR involving pedaling on bicycle had additional effects, such as improvement in fitness levels of patients with traumatic brain injury. This approach enhanced brain activation and improved cognitive processes [27]. A study [14] involving stroke patients showed that VR training significantly improved both visual and auditory attention after the treatment. Similar to the results of our study, long-term memory, such as those measured by verbal and visual learning tests, was also improved after the treatment in the VR training group. The natural environment provided on background screens in VR programs helped patients to adapt to the outside environment when they were discharged from the hospital. The various kinds of backgrounds provided are stimulating and interesting, which can help patients improve their visuospatial perception. The continuous visual and auditory stimuli provided through VR programs drawing patients' attention helped patients' concentration. The VR system has been used for patients with unilateral neglect. It was reported to induce attention to the contralateral visual site by giving patients cues in the contralateral visual site [13].

Improved attention can enhance the process of thinking and the storage and retrieval of memory, which helps patients regain their cognitive functions. In addition, full visual and auditory attentions are needed to accomplish tasks, which could trigger improvements in overall cognitive functions of patients. Since loss of attention could interfere with the recovery of other cognitive functions, cognitive treatments focusing on improvements in attention are needed for brain-injured patients with cognitive dysfunction [14].

Along with attention, the results of our study showed that many other categories of CNTs had significant improvement after the treatment in both groups. The rehabilitation programs given to the two groups were useful 
for improving overall cognitive functions. Our study also showed that activities of daily living were improved significantly after the treatment in both groups. This improvement may be due to repetitive stimuli given by training to both groups. In addition, improved attention might have enabled patients to be more engaged when performing activities of daily living.

There are several limitations to our study. First, our sample size was small. Studies with large sample sizes are needed to determine how much the VR training could improve cognitive function in patients with brain tumor. Second, we could not perform VR training alone. We had to combine these treatments because we could not allow the usual cognitive treatment with computer-assisted cognitive training of participants to be interrupted. Patients in the VR group received both computer-assisted cognitive rehabilitation and VR training. Therefore, it may be difficult to conclude that significant improvement of cognitive function in brain tumor patients was due to the effect of VR alone. Therefore, the sole effect of VR training in overall cognitive treatment remains unclear. A controlled study with only VR training should be given to identify its effect on cognitive function. Third, we could not distinguish how VR improved cognitive impairment according to brain tumor type. In addition, we could not conclude how it affected cognitive function according to brain tumor location. Further studies regarding these limitations should be undertaken in the future.

In conclusion, significant improvements in visual and auditory attention and short-term visuospatial memory were observed when VR and computer-assisted cognitive rehabilitation were given to brain tumor patients with cognitive dysfunction. However, it is difficult to conclude the sole action of VR training on cognitive function. Nonetheless, VR training has additional beneficial effects on cognitive improvement when it is combined with computer-assisted cognitive rehabilitation. Further randomized controlled studies with large sample sizes are needed to investigate how VR training improves cognitive impairment according to brain tumor type and location.

\section{CONFLICT OF INTEREST}

No potential conflict of interest relevant to this article was reported.

\section{REFERENCES}

1. Gehring K, Sitskoorn MM, Aaronson NK, Taphoorn MJ. Interventions for cognitive deficits in adults with brain tumours. Lancet Neurol 2008;7:548-60.

2. Shaw EG, Rosdhal R, D'Agostino RB Jr, Lovato J, Naughton MJ, Robbins ME, et al. Phase II study of donepezil in irradiated brain tumor patients: effect on cognitive function, mood, and quality of life. J Clin Oncol 2006;24:1415-20.

3. Pichierri G, Wolf P, Murer K, de Bruin ED. Cognitive and cognitive-motor interventions affecting physical functioning: a systematic review. BMC Geriatr 2011; 11:29.

4. dos Santos Mendes FA, Pompeu JE, Modenesi Lobo A, Guedes da Silva K, Oliveira Tde P, Peterson Zomignani A, et al. Motor learning, retention and transfer after virtual-reality-based training in Parkinson's disease: effect of motor and cognitive demands of games: a longitudinal, controlled clinical study. Physiotherapy 2012;98:217-23.

5. Cherniack EP. Not just fun and games: applications of virtual reality in the identification and rehabilitation of cognitive disorders of the elderly. Disabil Rehabil Assist Technol 2011;6:283-9.

6. Yip BC, Man DW. Virtual reality-based prospective memory training program for people with acquired brain injury. NeuroRehabilitation 2013;32:103-15.

7. Hanten G, Cook L, Orsten K, Chapman SB, Li X, Wilde EA, et al. Effects of traumatic brain injury on a virtual reality social problem solving task and relations to cortical thickness in adolescence. Neuropsychologia 2011;49:486-97.

8. Wang M, Reid D. Using the virtual reality-cognitive rehabilitation approach to improve contextual processing in children with autism. ScientificWorldJournal 2013;2013:716890.

9. Hofmann M, Rosler A, Schwarz W, Muller-Spahn F, Krauchi K, Hock C, et al. Interactive computer-training as a therapeutic tool in Alzheimer's disease. Compr Psychiatry 2003;44:213-9.

10. Davidsdottir S, Wagenaar R, Young D, Cronin-Golomb A. Impact of optic flow perception and egocentric coordinates on veering in Parkinson's disease. Brain 2008;131(Pt 11):2882-93.

11. Werner P, Rabinowitz S, Klinger E, Korczyn AD, Jos- 
man N. Use of the virtual action planning supermarket for the diagnosis of mild cognitive impairment: a preliminary study. Dement Geriatr Cogn Disord 2009;27:301-9.

12. Lee SJ, Chun MH. Combination transcranial direct current stimulation and virtual reality therapy for upper extremity training in patients with subacute stroke. Arch Phys Med Rehabil 2014;95:431-8.

13. Kim J, Kim K, Kim DY, Chang WH, Park CI, Ohn SH, et al. Virtual environment training system for rehabilitation of stroke patients with unilateral neglect: crossing the virtual street. Cyberpsychol Behav 2007;10:7-15.

14. Kim BR, Chun MH, Kim LS, Park JY. Effect of virtual reality on cognition in stroke patients. Ann Rehabil Med 2011;35:450-9.

15. Taphoorn MJ, Klein M. Cognitive deficits in adult patients with brain tumours. Lancet Neurol 2004;3:15968.

16. Weitzner MA, Meyers CA. Cognitive functioning and quality of life in malignant glioma patients: a review of the literature. Psychooncology 1997;6:169-77.

17. Anderson SW, Damasio H, Tranel D. Neuropsychological impairments associated with lesions caused by tumor or stroke. Arch Neurol 1990;47:397-405.

18. Meyers CA, Smith JA, Bezjak A, Mehta MP, Liebmann J, Illidge $T$, et al. Neurocognitive function and progression in patients with brain metastases treated with whole-brain radiation and motexafin gadolinium: results of a randomized phase III trial. J Clin Oncol 2004;22:157-65.

19. Holden MK. Virtual environments for motor rehabili- tation: review. Cyberpsychol Behav 2005;8:187-219.

20. Zelinski EM, Reyes R. Cognitive benefits of computer games for older adults. Gerontechnology 2009;8:22035.

21. Sveistrup H. Motor rehabilitation using virtual reality. J Neuroeng Rehabil 2004;1:10.

22. Kizony R, Raz L, Katz N, Weingarden H, Weiss PL. Video-capture virtual reality system for patients with paraplegic spinal cord injury. J Rehabil Res Dev 2005;42:595-608.

23. You SH, Jang SH, Kim YH, Hallett M, Ahn SH, Kwon $\mathrm{YH}$, et al. Virtual reality-induced cortical reorganization and associated locomotor recovery in chronic stroke: an experimenter-blind randomized study. Stroke 2005;36:1166-71.

24. Yong Joo L, Soon Yin T, Xu D, Thia E, Pei Fen C, Kuah $\mathrm{CW}$, et al. A feasibility study using interactive commercial off-the-shelf computer gaming in upper limb rehabilitation in patients after stroke. J Rehabil Med 2010;42:437-41.

25. Kim MY, Lee KS, Choi JS, Kim HB, Park CI. Effectiveness of cognitive training based on virtual reality for the elderly. J Korean Acad Rehabil Med 2005;29:42433.

26. Sanchez-Vives MV, Slater M. From presence to consciousness through virtual reality. Nat Rev Neurosci 2005;6:332-9.

27. Grealy MA, Johnson DA, Rushton SK. Improving cognitive function after brain injury: the use of exercise and virtual reality. Arch Phys Med Rehabil 1999;80:661-7. 